\title{
Which Emotion Regulation Strategies are Most Associated with Trait Emotion Dysregulation? A Transdiagnostic Examination
}

\section{Now Accepted at the Journal of Psychopathology and Behavioral Assessment}

\author{
Alexander R. Daros ${ }^{1}$ and Anthony C. Ruocco ${ }^{1,2,3}$ \\ ${ }^{1}$ Centre for Addiction and Mental Health, Toronto, Canada \\ ${ }^{2}$ Department of Psychology, University of Toronto Scarborough \\ ${ }^{3}$ Department of Psychological Clinical Science, University of Toronto
}

\section{Author Note}

Alexander R. Daros (iD https://orcid.org/0000-0001-9438-4524

Anthony C. Ruocco (iD https://orcid.org/0000-0002-1942-7181

Correspondence concerning this article should be addressed to Alexander R. Daros, Campbell Family Mental Health Research Institute, Centre for Addiction and Mental Health, 33 Russell Street, Suite 1027, Toronto, Ontario, Canada, M5S 2S1. Email: daros.alexander@gmail.com

\section{Declarations}

Conflicts of Interest/Competing Interests: There are no conflicts of interest or financial disclosures to declare.

Availability of data and material: Consent to deposit our dataset online was not included in the informed consent procedures during data collection.

Ethics approval: This study received an institutional review board (IRB) ethics approval before data collection began.

Funding: ARD was supported by a Doctoral Canada Graduate Scholarship from the Social Sciences and Humanities Research Council of Canada. ACR was supported by an Early Researcher Award (ER14-10-185) from the Province of Ontario's Ministry of Research and Innovation, New Investigator Salary Award (MSH130177) from the Canadian Institutes of Health Research, and Research Excellence Faculty Scholars Award from the University of Toronto Scarborough.

Acknowledgements: Thoughtful comments and feedback were provided by Shayan Asadi, Hadassah Beggs-Hebert, and Sasha Allen. 


\begin{abstract}
Although definitions of emotion dysregulation infer difficulties in selecting and implementing emotion regulation (ER) strategies, surprisingly few studies have examined the relationship between trait emotion dysregulation and a wide range of specific ER strategies. The present study used a data-driven approach to assess trait- and state-related ER strategy use in 99 women (aged 18-55) recruited from the community with varying levels of trait emotion dysregulation. Participants completed self-report questionnaires assessing habitual ER strategy implementation and self-ratings of ER strategy use in vivo during negative mood inductions. Principal components analysis revealed four self-report questionnaire-based and three moodinduction-based groupings comprising both optimal and suboptimal strategies. After adjusting for demographic and clinical variables, results from self-report questionnaires indicated that trait emotion dysregulation was significantly associated with higher endorsements of suboptimal strategies in two groupings (e.g., self-criticism, rumination, and social withdrawal; catastrophizing and blaming others) and lower endorsements of optimal ER strategies in one grouping (e.g., cognitive reappraisal and problem solving). In the context of mood induction, trait emotion dysregulation was significantly associated with higher endorsements of suboptimal ER strategies from one cluster only (e.g., expressive suppression, thought avoidance, and selfcriticism). Such transdiagnostic, data-driven approaches can uncover how the application of specific ER strategies both habitually and during negative mood states is associated with trait emotion dysregulation.
\end{abstract}

Keywords: emotion regulation; coping strategies; emotion dysregulation; principal components analysis 


\section{Which Emotion Regulation Strategies are Most Associated with Trait Emotion Dysregulation? A Transdiagnostic Examination}

Emotion dysregulation is a transdiagnostic symptom dimension underlying many forms of psychopathology (Beauchaine, 2015) and a prominent treatment target in psychological interventions (Sloan et al., 2017). Here, we define emotion dysregulation as the consequence of using fewer effective, and more ineffective, strategies to regulate negative emotions leading to the persistence of undesired negative emotional states over time (Gross \& Jazaieri, 2014; Linehan, 2014), which increases the risk for different mental disorders. There is interest in understanding how self-report assessments of emotion dysregulation converge with emotional experiences in daily life, but thus far research has mostly focused on trait- and state-ER strategy convergence (Koval et al., submitted for publication). Understanding the trait- and state-ER strategies used by individuals with varying trait levels of emotion dysregulation-regardless of their clinical diagnostic status - could reveal new targets of interventions that may serve a broader population of individuals with psychopathology. The present study uses an existing dataset to provide a preliminary assessment of trait- and state-ER (and broader coping) strategy use among women with varying levels of trait emotion dysregulation.

Definitions of emotion dysregulation allude to detriments in the implementation or selection of ER strategies. From an affective science perspective, emotion dysregulation is hypothesized to result from using ER strategies that are poorly matched to the context in which

they are used and/or not using ER strategies when it would be helpful to do so (Gross \& Jazieri, 2014). Others define emotion dysregulation as a pattern of emotional experiences and/or expression that interferes with goal-directed behavior (Beauchaine, 2015) or the persistence of undesired emotional states after effortful ER attempts are made (Cole \& Hall, 2008; Linehan, 
2014). The Difficulties in Emotion Regulation Scale (DERS; Gratz \& Roemer, 2004) is often used to assess emotion dysregulation using a multidimensional model consisting of difficulties in accepting negative emotional experiences, poor emotional awareness and clarity, difficulties engaging in goal-directed and non-impulsive behaviors, and accessing situationally-appropriate ER strategies. ${ }^{1}$ The DERS assesses these dimensions using dispositional tendencies, with instructions asking participants to rate their average or typical experiences, similar to trait-like characteristics that endure over time. Emotion dysregulation measured by the DERS is significantly higher in individuals with psychiatric diagnoses (e.g., borderline personality disorder [BPD], post-traumatic stress disorder) compared to non-clinical controls and is significantly associated with greater psychiatric symptoms, such as depression, anxiety, substance use, and dysregulated eating (see Gratz, Forbes, Wheeless, Richmond, \& Tull, 2020, for review). In the present study, we therefore operationalize the DERS total score as a trait symptom dimension of emotion dysregulation underlying psychopathology.

The DERS does not measure the relative endorsement frequency of specific ER strategies (e.g., cognitive reappraisal), however. Other research attempts to examine emotion dysregulation by studying the ER strategy endorsements in clinical samples consisting of different psychiatric diagnoses (e.g., major depressive disorder, generalized anxiety disorder) or their associations with psychiatric symptoms, such as depression or anxiety measured dimensionally (Aldao, Nolen-Hoeksema, \& Schweizer, 2010; Liu \& Thompson, 2017; Salters-Pedneualt, Roemer, Tull, Rucker, \& Mennin, 2006). Results generally indicate that individuals with various psychiatric

\footnotetext{
${ }^{1}$ The original DERS emphasizes responses to negative emotions, asking participants to often think of times "When I am upset..." and prompting negative affective states such as "ashamed", "guilty", and "irritated" suggesting an emphasis on emotion dysregulation in response to negative emotional experiences. Alternate versions address emotion dysregulation in response to positive emotions (Weiss, Gratz, \& Lavender, 2015) and current state- versus trait-like experiences in response to negative emotions (Lavender, Tull, DiLillo, Messman-Moore, \& Gratz, 2017).
} 
conditions, or greater symptoms of mental disorder, self-report using more faulty strategies to regulate negative emotions compared to healthy individuals or those with fewer psychiatric symptoms. Psychiatric groups appear more similar than different in this regard (e.g., Svaldi et al., 2012a), possibly due to shared underlying symptom dimensions around the experience of negative emotions and subsequent ER strategy use. Collectively, the literature on ER strategy use and emotion dysregulation support transdiagnostic approaches that go beyond the limitations of within-diagnosis heterogeneity and diagnostic comorbidity that are inherent to a categorical diagnostic system of psychiatric classification (see Kotov et al., 2017).

Few studies comprehensively assess multiple ER strategies in a single research design, instead focusing on one or a handful of well-defined strategies such as rumination, experiential avoidance, and suppression. Given evidence that flexible use of multiple ER strategies can contribute to mental and emotional well-being (Bonanno \& Burton, 2013) and that use of typically more effective ER strategies can mitigate the detrimental impact of generally less effective ER strategies (e.g., Aldao \& Nolen-Hoeksema, 2012), researchers have attempted to measure an individual's larger repertoire of ER strategies and the association with different forms of psychopathology (e.g., Blanke et al., 2019; De France \& Hollenstein, 2017; DixonGordon, Aldao, \& De Los Reyes, 2015). Studies also tend to combine ER strategies based on their putatively adaptive (e.g., problem-solving, cognitive reappraisal) or maladaptive (e.g., suppression, rumination) effects, with the former negatively and the latter positively associated with psychopathology (Aldao et al., 2010). Though this relationship appears straightforward on the surface, there are some diverse findings that are important to consider. For example, cognitive reappraisal is not always successful at modulating emotions, nor is it always adaptive across contexts (Ford \& Troy, 2019). In those with BPD, experience-sampling and laboratory- 
based mood inductions have shown that expressive suppression and avoidance tend to have more beneficial emotional effects in the short-term (e.g., lower urges to engage in impulsive behaviors), whereas acceptance is associated with short-term emotional consequences (e.g., increased urge to engage in self-injury and higher subjective distress; Chapman et al., 2009; Pistorello et al., 2015; Svaldi et al., 2012b). Such findings have led to the assertion that contextual factors (e.g., situational, such as social context, and personal, such as beliefs about emotions) contribute to the differential use of ER strategies, and that the flexibility in using different strategies across contexts is more predictive of well-being and psychopathology (Aldao, 2013). The present study therefore measured multiple ER strategies and made no a priori assumptions about the proposed adaptiveness of different strategies.

At the same time, other researchers have proposed data-driven approaches to group ER strategies into more manageable groupings that function similarly in response to an emotional stressor. The most comprehensive analysis to date reviewed 10 ER strategies assess via selfreport questionnaires (Naragon-Gainey, McMahon, \& Chacko, 2017). Three groupings were found: disengagement ER strategies characterized by attempts to avoid, inhibit, or shift focus away from an emotionally relevant situation (e.g., distraction, experiential avoidance, expressive suppression); engagement ER strategies characterized by attempts to actively engage with an emotional experience or material (e.g., problem solving, mindfulness, cognitive reappraisal); and aversive cognitive perseveration ER strategies characterized by difficulty disengaging from negative cognitions (e.g., worry, rumination). Although these groupings should be relatively consistent across studies using self-report methods, results may differ slightly based on the specific sample or methods involved in each study. For example, one previous study in college students found two groupings from eight ER strategies (Daros et al., 2020). In addition, trait 
emotion dysregulation at baseline was positively associated with a higher likelihood of endorsing disengagement versus engagement ER strategies in daily life during periods of high negative affect. The present study attempts to extend these findings by observing the convergence between trait emotion dysregulation and trait and state measures of ER using data from five separate self-report questionnaires and a laboratory-based mood induction procedure directly after which participants self-reported their ER strategy use. Data-driven techniques were used to reduce the number of ER strategies assessed into manageable groupings.

Finally, evidence for differential associations between facets of emotion dysregulation as assessed by the DERS (i.e., non-acceptance) and ER strategy use exist. Controlling for other facets, greater non-acceptance of negative emotions is associated with endorsing a higher proportion of disengagement versus engagement ER strategies during moments of high negative affect (Daros et al., 2020), as assessed by experience sampling methods. Moreover, difficulties with impulsivity and accessing ER strategies were associated with more ER strategy attempts. Difficulties with impulsivity during negative moods also differentially predicted greater purging frequency and lifetime non-suicidal self-injury - two behavioral mechanisms of ER — in a large heterogenous eating disorder sample (Pisetsky, Haynos, Lavender, Crow, \& Peterson, 2017). Thus, examining relationships between facets of trait emotion dysregulation and ER strategies were also considered in the present study.

The previous works were concerned with determining the differences in ER strategies between clinical diagnostic groups. There we found that a BPD group endorsed higher rates of maladaptive ER strategies and lower rates of effective ER strategies compared to healthy controls on both self-report measures (Daros, Guevara, Uliaszek, McMain, \& Ruocco, 2018a) and immediately following negative mood induction (Daros et al., 2018b). The BPD group did 
not differ from those with depressive and/or anxiety disorders on either assessment; however, in the first study, the BPD group endorsed a greater frequency of harmful regulatory behaviors (e.g., self-injury, eating binges) and in the second study, they reported higher perceived difficulty in regulating emotions, supporting higher levels of emotion dysregulation in BPD. The present study differs in several ways. First, we studied the relationships of ER strategies with trait emotion dysregulation regardless of diagnostic group. Second, we comprehensively assessed self-reported habitual ER strategies (including additional measures not already analyzed) alongside in vivo ratings during mood inductions; therefore, the multi-method nature of the data provides information about trait- and state-related ER strategy use and clarifies relationships with trait emotion dysregulation. Third, the present analysis takes a data-driven approach to grouping ER strategies rather than assigning them into pre-defined categories based on presumed adaptiveness, which can vary depending on situational and personal contextual factors.

Given previous findings on the structure of common ER strategies, we expected to find at least three ER strategy groupings closely aligning to disengagement, engagement, and cognitive perseveration on both the self-report questionnaires and endorsements made in vivo following mood induction. We then expected trait emotion dysregulation to be positively associated with endorsements of disengagement and cognitive perseveration ER strategies and negatively associated with engagement ER strategies. We then explored whether the same patterns existed for facets of trait emotion dysregulation (i.e., non-acceptance, difficulties with impulsivity), as measured by the DERS. Findings from this study have potential to highlight how specific habitual and mood-induction-based endorsements of ER strategies contribute to trait emotion dysregulation in a sample of women with varying levels of psychopathology, which may aid further transdiagnostic-focused clinical research and inform treatment considerations. 


\section{Method}

\section{Participants}

The previous work involved the recruitment of three target diagnostic groups from 20152016: women with current BPD; women with a current depressive and/or anxiety disorder; and healthy women with no current or previous diagnosis. Because of our difficulty in recruiting men with BPD from the community (see Ruocco et al., 2019) and notable sex and gender differences with respect to emotional experience and ER reported in the literature (e.g., Kring \& Gordon, 1998; McRae, Ochsner, Mauss, Gabrieli, \& Gross, 2008), we elected to recruit women exclusively. We recruited 101 women (aged 18-55) from the community who were English speaking and able to provide written informed consent. Online postings were used (e.g., Kijiji, Facebook) to seek individuals who had seen a doctor or a mental health professional for depression, anxiety, and/or BPD; all participants were screened by telephone before attending the study. Exclusion criteria for all groups included any psychotic or bipolar I disorder, neurological illness, neurodevelopmental disorder, and current alcohol or non-alcohol substance dependence. The exclusion criteria extended from the original work and were implemented to reduce potential confounds on cognitive and physiological tasks that were delivered in a laboratory (i.e., nonhospital) setting. Participants in the healthy group were not excluded if they had a history of psychotherapy. Participants in the clinical groups were not excluded if they were currently or previously completing psychotherapy or pharmacological treatments.

Following participation, two women were excluded from the present analyses because they did not complete the DERS, leaving 99 participants with the necessary self-report measures completed. During the mood induction procedure, one participant requested discontinuation and was removed from analysis. Two other participants were presented with an incorrect video due 
to experimenter error, thus these data were simply treated as missing (the other videos they watched were retained). Participants were compensated up to $\$ 50$ for their participation in the larger study (more details can be found in our previous studies). The University of Toronto Research Ethics Board approved the study.

\section{Diagnostic Assessments}

Participants were assessed using the Structured Clinical Interview for DSM-IV Axis I disorders - Patient Edition (First, Spitzer, Gibbon, \& Williams, 2002). The modules for mood, bipolar, psychotic, substance use, and anxiety disorders were used, but due to time constraints, we were unable to assess eating or other classes of psychiatric disorders. Interviews were completed by Master's- and doctoral-level interviewers under supervision of a licensed clinical psychologist (A.C.R). Interviewers prepared diagnostic reports that were discussed at "bestestimate" diagnostic meetings to establish consensus (Klein, Ouimette, Kelly, Ferro, \& Riso, 1994). The present sample had the following diagnostic characteristics: Major Depressive Disorder, Current/Past (N=28/29); Dysthymic Disorder, Current (N=1); Bipolar II Disorder, Current (N=1); Alcohol Abuse or Dependence, Past (N=22); Substance Abuse or Dependence, Past (N=13); Panic Disorder with or without Agoraphobia, Current/Past (N=17/10); Agoraphobia, Current/Past (N=2/1); Social Phobia, Current/Past (N=28/8); Specific Phobia, Current (N=1); Obsessive Compulsive Disorder, Current/Past (N=2/3); Post-Traumatic Stress

Disorder, Current/Past (N=10/10); and Generalized Anxiety Disorder, Current $(\mathrm{N}=18){ }^{2}$

Interviewers also used the BPD module of the Structured Interview for DSM-IV

Personality (Pfohl, Blum, \& Zimmerman, 2009) to assess the presence of BPD symptoms.

According to the test manual, each symptom is assessed on a 4 -point scale: $0=$ not present, $1=$

\footnotetext{
2 Two participant diagnostic reports could not be completed because of incomplete information and were coded as Diagnosis Deferred.
} 
subthreshold, $2=$ present, and $3=$ present and associated with subjective distress and/or functional impairment. Participants had to meet 5 or more current criteria in the past 5 years for the BPD diagnosis to be applied. In the current sample participants met an average of 2.88 symptom criteria $(S D=3.10)$ and 32 participants met the diagnostic threshold for BPD.

\section{Trait Measure of Emotion Dysregulation}

Each participant included in the study completed the DERS (Gratz \& Roemer, 2004), which consists of 36 items rated on a 1 (almost never, 0-10\%) to 5 (almost always, 90-100\%) scale, with higher scores indicative of greater difficulties regulating emotions. The DERS is an established measure of emotion dysregulation used to assess "maladaptive responses to emotions" (Gratz et al., 2020) compared to other measures that typically assess emotional responses themselves (e.g., emotional reactivity and perceived intensity). Thus far, the DERS remains the only measure associated with objective measures of emotion dysregulation assessed via behavioral, physiological, and neuroimaging methods. Total scores of 80-100 are often reported by individuals with emotional disorders, with scores above 110 often found in those with BPD (Daros et al., 2018a; Svaldi et al., 2012a). Along with a total score, six subscale scores can be computed: (a) non-acceptance of negative emotions (Non-acceptance, 6 items); (b) difficulties engaging in goal-directed behaviors (Goals; 5 items); (c) difficulties refraining from impulsive behaviors during negative emotions (Impulsivity; 6 items); (d) lack of emotional awareness (Awareness; 6 items); (e) lack of emotional clarity (Clarity; 5 items); and (f) limited access to situationally appropriate strategies (Strategies; 8 items). In the current study, internal consistencies for the DERS total and each subscale were either acceptable or higher $(\alpha \mathrm{s}>.79)$, which was similar to the initial validation ( $\alpha$ s $>.80$; Gratz \& Roemer, 2004). Evidence for testretest reliability for the DERS total score was high $(r=.88)$ over one to two months in the initial 
validation (subscales $r \mathrm{~s}=.57-.89$; Gratz \& Roemer, 2004), supporting the trait-like quality of emotion dysregulation as measured by the DERS.

\section{Symptom Measures}

The Depression, Anxiety, and Stress Scale (DASS-42; Lovibond \& Lovibond, 1995) was used to assess depression, anxiety, and stress symptoms over the past two weeks. The internal consistency of the depression and anxiety subscales were good-to-excellent ( $\alpha$ s $>.89$ ).

Participants also completed the first 12 items of the Borderline Evaluation of Severity over Time (BEST; Pfohl et al., 2009), a self-report measure of BPD symptom severity over the past two weeks. The internal consistency of the total score was excellent $(\alpha=.90)$.

\section{Trait Measures of ER Strategies}

Participants completed five separate self-report measures to assess habitual ER strategy use and coping: The White Bear Suppression Inventory (WBSI; Wegner \& Zanakos, 1994), the Ruminative Response Style (RRS; Treynor, Gonzalez, \& Nolen-Hoeksema, 2003), the Multidimensional Experiential Avoidance Questionnaire (MEAQ; Gamez, Chmielewski, Kotov, Ruggero, \& Watson, 2011), the Cognitive Emotion Regulation Questionnaire (CERQ; Garnefski \& Kraaij, 2007), and the Coping Strategies Inventory (Tobin, Holroyd, Reynolds, \& Wigal, 1989). Because none of these measures comprehensively assess the wide array of cognitive and behavioral ER strategies that have been identified in the affective science research literature (see Aldao \& Dixon-Gordon, 2014), the present study considered all 25 of the resulting variables computed from these measures with the intention of using principal components analysis (PCA) to reduce them to a more manageable number suitable for statistical analysis. ${ }^{3}$

\footnotetext{
${ }^{3}$ These measures were chosen because of an interest in how individuals downregulate negative emotional experiences associated with depression, anxiety, and BPD. We also chose measures that would allow us to assess a diverse range of strategies and coping tactics while also achieving adequate coverage (e.g., full scale or subscale) for the most commonly researched emotion regulation strategies from the affective science literature: cognitive
} 
The WBSI is a 15-item unidimensional measure to assess suppression of unwanted thoughts, with the total score reflecting an individual's tendency to engage in thought suppression. The RRS is a 22-item unidimensional measure of repetitive, ruminative thought patterns in reaction to negative events, with the total score typically used as a general indication of rumination. The MEAQ is a 62-item measure of experiential avoidance and a tendency to avoid negative experiences encompassing 6 subscales: behavioral avoidance, distress aversion, distress endurance, repression/denial, distraction/suppression, and procrastination. The CERQ is a widely used 36-item measure assessing nine different cognitive strategies to regulate emotions: positive refocusing, positive reappraisal, putting into perspective, refocus on planning, acceptance, blaming others, self-blame, rumination, and catastrophizing. The CSI is a 72-item inventory that assesses a eight types of coping/ER strategies in response to negative life events: problem-solving, cognitive reappraisal, emotional expression, social support, problem avoidance, wishful thinking, self-criticism, and social withdrawal. All items were presented in their original format with their corresponding Likert rating scales.

The WBSI, RRS, and MEAQ produced excellent internal consistency in one of our previous studies with a similar sample ( $\alpha \mathrm{s}>.89$; Daros et al., 2018a). The temporal stability of the WBSI and RRS has been reported as .69 over three weeks to three months (Wegner \& Zanakos, 1994) and .60 over 1 year (Treynor et al., 2003); test-retest reliability has not been evaluated for the MEAQ (see Gamez et al., 2011). In its initial validation, each CERQ subscale produced an internal consistency above .75 and test-retest reliabilities were between .48 and .65 over a 1-year period (Garnefski \& Kraaij, 2007). In its initial validation, each subscale from the 
CSI produced an internal consistency above .72 and the test-retest reliabilities were between .68 and .83 over two weeks (Tobin et al., 1989).

\section{State Measures of ER Strategies during Mood Induction}

The mood induction procedure included one neutral and three negative videos that were all four minutes in length. Here, only the negative videos were considered as it allowed for repeated within-person measurements of ER strategy endorsements. The negative videos depicted (a) a scene of domestic abuse between a man and a pregnant woman, where afterwards it is suggested that she loses the baby; (b) a funeral scene in which a young girl struggles to accept the death of her friend; and (c) a scene involving sexual assault by a police officer while interrogating a woman, with her husband watching. Participants completed a measure of baseline mood using the Positive and Negative Affect Scale (PANAS; Watson, Clark, \& Tellegen, 1988), which was then assessed again after each negative video, along with ratings of nine ER strategies written in lay terms (see below) and ratings of induced discrete emotions (e.g., disgust, sadness, fear, anger, happiness, and surprise). Using the current sample, each video was found to significantly increase negative affect on the PANAS compared to baseline using paired-samples t-tests, $t \mathrm{~s}>5.83, p \mathrm{~s}<.0001$. Compared to the neutral video, the three negative videos also elicited higher ratings of negative emotions (i.e., sum of individual ratings for fear, sadness, disgust, and anger; $t \mathrm{~s}>3.60, p \mathrm{~s}<.001)$. These clips were presented on a computer monitor using links to a private YouTube account embedded into a Qualtrics survey that was locked to a countdown timer so that the participant could not stop the video nor move forward until it was finished. Participants were instructed to maintain their visual attention on the computer monitor, avoid closing their eyes as much as possible, and were told that the goal of the study was to simply immerse themselves into each video. The entire mood induction procedure lasted 
approximately 40 minutes and participants were shown de-stressing (i.e., comedy or amusing animal) videos and could listen to music to aid in mood correction.

Immediately after each negative video, participants were asked to rate the extent to which they used a series of uninstructed ER strategies to manage their emotional response during the induction, written in lay-person descriptions: experiential avoidance (closing or averting your eyes), acceptance (allowing or accepting your feelings), cognitive reappraisal (thinking of the situation differently to change how you feel), problem-solving (coming up with ideas to change the situation or fix the problem), thought suppression (pushing down feelings or putting them out of mind), expressive suppression (hiding feelings from others), self-criticism (criticizing yourself for your feelings), thought avoidance (avoiding thinking about it), and rumination (worrying or ruminating about the situation). Participants rated each strategy on a Likert scale ranging from 1 (not at all) to 4 (a lot) based on a previous study (Aldao \& Nolen-Hoeksema, 2013). The mean ratings on these nine strategies collapsed over three videos were subjected to PCA.

Principal Components Analysis. Zero-order correlations between variables were not too low or too high ( $r$ s between .10 and .75), which supported the use of PCA (Field, 2013). A direct oblimin rotation and Kaiser normalization was used for both subsets of data, allowing the resulting components to be correlated with each other. Considering the sample size, factor loadings below .51 were suppressed and only variables with an internal consistency greater than or equal to .79 in the present sample were used to improve the resulting output of the analysis (Field, 2013). The resultant scores created by the analyses for each participant were saved for simultaneous entry in regression models, which is an effective method to reduce multicollinearity. Linear regression models were then used to estimate trait emotion dysregulation as a dependent variable using the resultant component scores for each participant and their clinical 
and demographic variables as control predictors. Age and years of education were continuous predictors whereas ethnicity was entered as a binary predictor $($ Caucasian $=1$; Other $=0)$.

For the self-report measures, the output produced four components (linear combinations of the inputted variables) with all eigenvalues greater than 1.64. The factor matrix is reproduced in Table 1 along with the internal consistency of each variable used to generate the output of the procedure. The Kaiser-Meyer-Olkin $(\mathrm{KMO}=.81)$ measure verified the sampling adequacy of the analysis (Hutcheson \& Sofroniou, 1999). Four variables were dropped due to low internal consistency prior to analysis and one variable was dropped because it did not load onto any resulting component (see Table 1). For the mood induction portion, the KMO (.75) measure verified the sampling adequacy of the analysis and only one variable was dropped because it did not load onto any component (see Table 2). The output produced three components, with all eigenvalues greater than 1.05. The factor matrix is reproduced in Table 2. The internal consistency of mean ratings for the nine strategies across the three negative videos was .79.

\section{Results}

\section{Sample Characteristics}

On average, the women were 28.27 years old $(S D=9.14)$ and had completed some university or college education (years of education: $M=14.68, S D=1.81$ ). The sample was racially/ethnically diverse: Caucasian (50.50\%), Asian/Southeast Asian (18.18\%), Mixed/Other (11.11\%), South Asian (9.09\%), Black (6.06\%), Latinx (3.03\%), and Arab (2.02\%). On average, participants reported a DERS total score of 92.64 out of a maximum of $180(S D=29.71$, Range =39-158; Median = 90.00) and the scores were pseudo-normally distributed, with no statistical outliers beyond +/- 2.0 SD. A frequency distribution of total scores according to the original recruitment groups is presented in Figure 1, demonstrating that the distribution was not biased in 
extreme ways. The average depression and anxiety scores were $13.31(S D=12.33$; Range $=0$ $40 ;$ Median $=11.00)$ and $8.81(S D=8.26 ;$ Range $=0-32 ;$ Median $=6.00)$ respectively, which both corresponded to mild symptoms. In addition, the average BEST score for BPD symptom severity was $10.67(S D=10.06$, Range $=0-35$; Median $=8.00)$. Trait emotion dysregulation was significantly associated with depression, anxiety, and BPD symptoms $(r s>.61, p s<.001)$.

\section{Component Analyses}

Self-Report Measures. The four emerging components explained $68.50 \%$ of the variance. Self-report component 1 (SRC1) largely subsumed the less putatively optimal strategies of selfcriticism, withdrawal, thought suppression, rumination, denial, and wishful thinking. SRC2 loaded onto the putatively more effective strategies of cognitive reappraisal, problem-solving, seeking social support, reframing, and tolerance of distress. SRC3 covered physical avoidance, distraction/suppression, and positive thinking. SRC4 concerned catastrophizing, blaming others, and negative evaluations of stress. SRC1 was negatively correlated with SRC2 $(r=-.33)$ and SRC3 $(r=-.18)$ and was positively associated with SRC4 $(r=.28)$. Additional inter-correlations between components were non-significant $(|r| \mathrm{s}<.14)$.

SRC1 $(r=.80)$ and SRC4 $(r=.47)$ were significantly positively correlated with trait emotion dysregulation, while SRC2 was negatively associated $(r=-.42)$. SRC3 was not significantly associated with trait emotion dysregulation $(r=.04)$. SRC1 and SRC4 were also significantly associated with depression, anxiety, and BPD symptoms ( $r$ s > .27), SRC2 was negatively associated with depression $(r=-.36)$ and BPD symptoms $(r=-.31)$, and SRC3 was not significantly associated with any clinical measures. A linear regression predicting trait emotion dysregulation from the four components while also controlling for demographic and clinical symptoms significantly improved prediction over a baseline model with demographic 
variables only (Table 3, upper portion). SRC1 and SRC4 were positive predictors of trait emotion dysregulation while SRC2 and years of education were negative predictors after controlling for demographic variables.

Mood Induction. The three emerging components explained $60.36 \%$ of the variance. Mood induction component 1 (MIC1) consisted of thought avoidance, suppression, and selfcriticism; MIC2 subsumed acceptance and rumination; and MIC3 covered the primarily more functional cognitive reappraisal and problem-solving strategies. MIC1 was negatively correlated with MIC3 $(r=-.25)$ but all other inter-correlations were non-significant $(|r| \mathrm{s}<.09)$, likely because of the weaker MIC2 component.

MIC1 $(r=.46)$ was positively associated with trait emotion dysregulation, while MIC3 was negatively associated $(r=-.15)$. MIC2 did not reach statistical significance $(r=.13)$ using a more stringent threshold of alpha (.01). MIC1 was positively associated with depression, anxiety, and BPD symptoms $(r \mathrm{~s}>.41)$, MIC3 was negatively associated with BPD symptoms only $(r=-$ .27 ), and MIC2 was not significantly associated with clinical measures. A linear regression predicting trait emotion dysregulation from the three components while also controlling for demographic and clinical symptoms significantly improved prediction over a baseline model with demographic variables only (Table 3, lower portion). However, only MIC1 remained a significant positive predictor of trait emotion dysregulation, whereas years of education remained the only significant negative predictor, after controlling for demographic variables.

\section{Ancillary Analyses}

To explore specific relationships between ER strategy clusters and facets of trait emotion dysregulation, correlations between the clusters and DERS subscales were computed using partial correlations that controlled for age, years of education, and ethnicity. As seen in Table 4, 
several patterns were revealed. SRC1 (self-criticism, withdrawal, thought suppression, wishful thinking, denial, and rumination strategies) was most strongly and significantly associated with all facets of trait emotion dysregulation, particularly non-acceptance and lack of access to situationally appropriate ER strategies. SRC2 (cognitive reappraisal, problem-solving, seeking social support, reframing, and overall distress tolerance) was significantly associated with most facets of trait emotion dysregulation, except non-acceptance and difficulties in goal-directed behavior during negative emotions. MIC1 (thought avoidance, suppression, and self-criticism) is most similar to SRC1 and was associated with most trait emotion dysregulation facets, except difficulties in emotional awareness. SRC4 (catastrophizing, blaming others, and negative evaluations of stress) was significantly associated with difficulties around access to strategies, goal-directed behavior, and impulsivity during negative emotions. MIC3 (cognitive reappraisal and problem-solving) had weak, non-significant associations with facets of trait emotion dysregulation, especially when the significance threshold was controlled.

\section{Discussion}

This study is unique in that it examines trait emotion dysregulation in a transdiagnostic manner (rather than comparing across clinical groups), simultaneously examines multiple ER strategies in a single research design, and utilizes a data-driven approach to cluster strategies derived from two methods assessing trait- and state-related ER strategy implementation. The most prominent ER strategies utilized by women with high trait emotion dysregulation were those that tend to be detrimental to mental health in the long-term and are associated with increased psychopathology as evidenced by the SRC1 and MIC1 groupings: self-criticism, withdrawal, social isolation, denial, wishful thinking, and rumination (Aldao et al., 2010; Naragon-Gainey et al., 2017). It is possible that lower distress tolerance and higher negative 
urgency (other dimensions associated with high trait emotion dysregulation) may increase motivation to end negative emotional experiences more quickly to limit negative emotional experiences with less cognitive effort (Jeffries et al., 2016; King et al., 2018). Given that SRC1 was highly associated with non-acceptance and difficulties selecting situationally appropriate ER strategies in our facet analyses, this may suggest that women with high trait emotion dysregulation have difficulties tolerating and engaging with negative emotional experiences.

Strategies that seem ineffective may have been learned as appropriate or adaptive in certain developmental contexts (e.g., hiding thoughts and feelings to prevent punishment; Linehan, 2014; Thompson, 2019). For example, first-degree family members of individuals with BPD tend to report intermediary levels of trait emotion dysregulation compared to non-familial healthy controls (Ruocco et al., 2019) and parents who report higher emotion dysregulation tend to invalidate their children's emotional experiences, which in turn predicts higher levels of emotion dysregulation in children (Buckholdt, Parra, \& Jose-Shields, 2014; Han \& Shaffer, 2013). It is possible that the high loadings of self-criticism and self-blame on SRC1, and their positive relationship to trait emotion dysregulation, may relate to how self-criticism can exacerbate the variability of negative emotions (Vansteelandt et al., 2019). Still, more research is needed to understand the reasons why ER strategies that seem ineffective on the surface are used more often by those with higher trait emotion dysregulation.

Women with higher levels of trait emotion dysregulation were also significantly less likely to endorse ER strategies associated with mental health benefits and fewer symptoms of psychopathology: cognitive reappraisal, problem-solving, seeking social support, reframing, and tolerance of distress (at least when measured via self-report questionnaires, SRC2). Strategies such as cognitive reappraisal tend to be more effortful because they involve engaging with the 
emotional stimulus (see Naragon-Gainey et al., 2017). This may be more challenging for people with high trait emotion dysregulation given other overlapping cognitive and emotional problems, similar to what is reported in major depressive disorder (Joorman \& Stanton, 2016). Other theories as to why optimal strategies are endorsed less often in high trait emotion dysregulation may relate to an individual's beliefs about specific ER strategies, their actual abilities or skill in modulating negative emotions, and the heightened intensity of emotional experience during exposure to negative stimuli (Yoon \& Rottenberg, 2020). Trait emotion dysregulation is also negatively correlated with mindfulness abilities which, in turn, may mediate greater thought avoidance (Prakash, Whitmoyer, Aldao, \& Schirda, 2017). SRC2 strategies were negatively associated with difficulties in emotional awareness and clarity (i.e., knowing what emotion one is feeling and distinguishing it from others) in our facet analyses. Those with more of these difficulties may have more challenges selecting situationally appropriate ER strategies and restrict themselves to less effective strategies to reduce emotional arousal (e.g., Vine \& Aldao, 2014). Collectively, more research is needed to determine the underlying mechanisms for reduced ER strategy effectiveness in individuals with higher trait emotion dysregulation.

Finally, women with higher trait emotion dysregulation also self-reported engaging in higher degrees of blaming others, catastrophizing, and evaluating distress in a more negative light. This grouping (SRC4) was also associated with a lack of access to situationally appropriate ER strategies when examining facets of trait emotion dysregulation. These findings fit with previous research on BPD demonstrating that trait emotion dysregulation is highly associated with repetitive thought processes (e.g., rumination, catastrophizing, and brooding; Selby, Anestis, Bender, \& Joiner, 2009), as well as anger rumination and interpersonal problems, which may lead to outbursts with others (e.g., Peters, Smart, \& Baer, 2015; Stepp et al., 2014). Specific 
evidence for the relationship between trait emotion dysregulation and "blaming others" appears sparse, although it is associated with expressions of anger (e.g., Martin \& Dahlen, 2005). As a specific subscale on the CERQ, "blaming others" is associated with psychopathology but much more weakly than other subscales (Garnefski \& Kraaji, 2007). People with high trait emotion dysregulation evaluate emotions more negatively (e.g., negative feelings are bad, they last forever, and control behavior; Veilleux et al., submitted for publication). Low perceived distress tolerance is associated with higher endorsements of suppression, avoidance, and rumination strategies (Jeffries et al., 2016), perhaps to avoid confronting or engaging with negative emotions in the present moment (see Leyro, Zvolensky, \& Bernstein, 2010).

Our PCA produced one additional self-report questionnaire component compared to a previous meta-analysis (Naragon-Gainey et al., 2017); however, we also had 15 additional variables. SRC1 and MIC1 most closely map onto the cognitive perseveration class, with overlap on rumination, worry, and thought suppression. The present study suggests that self-criticism, self-blame, denial, and wishful thinking may be additional cognitive perseveration strategies. SRC2 and MIC3 most closely map onto the engagement class, with cognitive reappraisal and problem-solving (as well as variants of these) the clearest overlapping ER strategies. The present study did not include a strong measure of acceptance/mindfulness; therefore, we were unable to replicate previous effects involving this strategy. SRC3 most closely aligns with the disengagement ER strategy grouping with overlap on distraction, behavioral avoidance, and experiential avoidance/suppression. SRC4 was a novel grouping, with strategies that were not examined in the previous meta-analysis. One other difference was that acceptance and rumination were in the same direction and separated on MIC2, whereas the previous metaanalysis suggested they might have loaded in the opposite direction and on MIC1. The reasons 
for this could relate to how the strategies were presented during the mood induction procedure and what participants interpreted them to mean. Because the current study avoided labeling these two strategies as antagonistic to each other (e.g., adaptive versus maladaptive), results indicated that endorsement ratings were more similar than different, at least during the mood induction procedure. These results are compelling evidence for data-driven approaches to grouping ER strategies, as it may be difficult to determine their common structure before data analysis; moreover, that structure may also depend on the sample assessed.

Although we had the benefit of a broad range of trait emotion dysregulation scores, limitations of this work include the relatively small sample size, restrictions to participation based on the presence of psychotic or substance use disorders, and to the ability to generalize these results to all women or outside the clinical characteristics of the current sample. Moreover, the restriction of the current sample to women was based on the previous work which was primarily conducted to examine differences in women with BPD compared to women with depressive and/or anxiety disorders and non-clinical healthy controls. There are plausible differences in the ways that men and women utilize ER strategies (e.g., Zlomke \& Hahn, 2010); therefore, the current study requires replication and extension to samples that include men. Considerations of ethnicity could also be improved, given this study only examined potential differences between Caucasian women and those of other ethnicities. Although two methods of assessment were used, they both relied on self-reflection to report emotional experiences, which may be difficult for some individuals. As noted above, people with higher (versus lower) trait emotion dysregulation may also exhibit lower emotional clarity and awareness, which may impact how they report their use of ER strategies. More broadly, this work is correlational in its design and we cannot draw conclusions about causality or the directionality of the effects, or 
whether a third variable may have influenced the results. Future research will be needed to address these limitations, extend them to more diverse demographic samples, and investigate alternative assessment methodologies.

Despite these limitations, the present study provides an initial, comprehensive investigation of the relationship between trait emotion dysregulation and specific ER strategies in the context of trait- and state-relevant assessment methods. Our findings suggest that women with higher levels of trait emotion dysregulation are most likely to use suboptimal strategies to regulate their emotions (i.e., self-criticism, social isolation, rumination, blaming others, and catastrophizing) and less likely to use generally more effective ones (i.e., cognitive reappraisal, problem-solving, seeking social support, and tolerance of distress), while controlling for demographic variables. These findings are in line with theories on the development of emotion dysregulation from a transdiagnostic clinical perspective (Linehan, 2014; Thompson, 2019) and may be used to understand how habitual and negative mood-state-related use of certain ER strategies contributes to psychopathology and vice versa (i.e., how psychopathology is maintained by ER strategy use). Further, these results could be used to understand how treatments work to reduce the use of suboptimal ER strategies and increase the implementation of more effective ones. 


\section{References}

Aldao, A. (2013). The future of emotion regulation research: Capturing context. Perspectives on Psychological Science, 8(2), 155-172. https://doi.org/10.1177/1745691612459518

Aldao, A., \& Dixon-Gordon, K. L. (2014). Broadening the scope of research on emotion regulation strategies and psychopathology. Cognitive Behaviour Therapy, 43(1), 22-33. https://doi.org/10.1080/16506073.2013.816769

Aldao, A., \& Nolen-Hoeksema, S. (2012). When are adaptive strategies most predictive of psychopathology? Journal of Abnormal Psychology, 121(1), 276-281. https://doi.org/10.1037/a0023598

Aldao, A., Nolen-Hoeksema, S., \& Schweizer, S. (2010). Emotion-regulation strategies across psychopathology: A meta-analytic review. Clinical Psychology Review, 30(2), 217-237. https://doi.org/10.1016/j.cpr.2009.11.004

Beauchaine, T. P. (2015). Future directions in emotion dysregulation and youth psychopathology. Journal of Clinical Child \& Adolescent Psychology, 44(5), 875-896. https://doi.org/10.1080/15374416.2015.1038827

Blanke, E. S., Brose, A., Kalokerinos, E. K., Erbas, Y., Riediger, M., \& Kuppens, P. (in press). Mix it to fix it: Emotion regulation variability in daily life. Emotion.

\section{https://doi.org/10.1037/emo0000566}

Bonanno, G. A., \& Burton, C. L. (2013). Regulatory flexibility: An individual differences perspective on coping and emotion regulation. Perspectives on Psychological Science, 8(6), 591-612. https://doi.org/10.1177/1745691613504116

Buckholdt, K. E., Parra, G. R., \& Jobe-Shields, L. (2014). Intergenerational transmission of emotion dysregulation through parental invalidation of emotions: Implications for adolescent internalizing and externalizing behaviors. Journal of Child and Family Studies, 23(2), 324332. https://doi.org/10.1007/s10826-013-9768-4 
Chapman, A. L., Rosenthal, M. Z., \& Leung, D. W. (2009). Emotion suppression in borderline personality disorder: An experience sampling study. Journal of Personality Disorders, 23(1), 29-47. https://doi.org/10.1521/pedi.2009.23.1.29

Cole, P. M., \& Hall, S. E. (2008). Emotion dysregulation as a risk factor for psychopathology. In T. P. Beauchaine, S. P. Hinshaw (Eds.), Child and adolescent psychopathology (pp. 265298). John Wiley \& Sons, Inc.: New Jersey.

Daros, A. R., Guevara, M. A., Uliaszek, A. A., McMain, S. F., \& Ruocco, A. C. (2018a). Cognitive emotion regulation strategies in borderline personality disorder: Diagnostic comparisons and associations with potentially harmful behaviors. Psychopathology, 51(2), 83-95. https://doi.org/10.1159/000487008

Daros, A. R., Williams, G. E., Jung, S., Turabi, M., Uliaszek, A. A., \& Ruocco, A. C. (2018b). More is not always better: Strategies to regulate negative mood induction in women with borderline personality disorder and depressive and anxiety disorders. Personality Disorders: Theory, Research, and Treatment, 9(6), 530-542. https://doi.org/10.1037/per0000296

Daros, A. R., Daniel, K. E., Boukhechba, M., Chow, P. I., Barnes, L. E., \& Teachman, B. A. (2020). Relationships between trait emotion dysregulation and emotional experiences in daily life: An experience sampling study. Cognition and Emotion, 34(4), 743-755. https://doi.org/10.1080/02699931.2019.1681364

De France, K., \& Hollenstein, T. (2017). Assessing emotion regulation repertoires: The regulation of emotion systems survey. Personality and Individual Differences, 119, 204215. https://doi.org/10.1016/j.paid.2017.07.018

Dixon-Gordon, K. L., Aldao, A., \& De Los Reyes, A. (2015). Repertoires of emotion regulation: A person-centered approach to assessing emotion regulation strategies and links to psychopathology. Cognition and Emotion, 29(7), 1314-1325. https://doi.org/10.1080/02699931.2014.983046 
Field, A. (2013). Discovering statistics using IBM SPSS statistics. Sage Publications: New York.

First, M., Spitzer, R., Gibbon, M., \& Williams, J. (2002). Structured Clinical Interview for DSMIV-TR, Research Version, Non-Patient Edition. New York, NY: New York State Psychiatric Institute.

Ford, B. Q., \& Troy, A. S. (2019). Reappraisal reconsidered: A closer look at the costs of an acclaimed emotion-regulation strategy. Current Directions in Psychological Science, 28(2), 195-203. https://doi.org/10.1177/0963721419827526

Gámez, W., Chmielewski, M., Kotov, R., Ruggero, C., \& Watson, D. (2011). Development of a measure of experiential avoidance: The Multidimensional Experiential Avoidance Questionnaire. Psychological Assessment, 23(3), 692-713. https://doi.org/10.1037/a0023242

Garnefski, N., \& Kraaij, V. (2007). The cognitive emotion regulation questionnaire. European Journal of Psychological Assessment, 23(3), 141-149. https://doi.org/10.1027/1015-

\section{$\underline{5759.23 .3 .141}$}

Gratz, K. L., Forbes, C. N., Wheeless, L. E., Richmond, J. R., \& Tull, M. T. (2020). Self-report assessment of emotion dysregulation. In T. P. Beauchaine \& S. E. Crowell (Eds.), The Oxford Handbook of Emotion Dysregulation (pp.395-409). Oxford University Press: New York.

Gratz, K. L., \& Roemer, L. (2004). Multidimensional assessment of emotion regulation and dysregulation: Development, factor structure, and initial validation of the difficulties in emotion regulation scale. Journal of Psychopathology and Behavioral Assessment, 26(1), 41-54. https://doi.org/10.1023/B:JOBA.0000007455.08539.94

Han, Z. R., \& Shaffer, A. (2013). The relation of parental emotion dysregulation to children's psychopathology symptoms: The moderating role of child emotion dysregulation. Child Psychiatry \& Human Development, 44(5), 591-601. https://doi.org/10.1007/s10578-012$\underline{0353-7}$ 
Hutcheson, G. D., \& Sofroniou, N. (1999). The multivariate social scientist: Introductory statistics using generalized linear models. Sage Publications: New York. https://doi.org/10.4135/9780857028075

Jeffries, E. R., McLeish, A. C., Kraemer, K. M., Avallone, K. M., \& Fleming, J. B. (2016). The role of distress tolerance in the use of specific emotion regulation strategies. Behavior Modification, 40(3), 439-451. https://doi.org/10.1177/0145445515619596

Joormann, J., \& Stanton, C. H. (2016). Examining emotion regulation in depression: A review and future directions. Behaviour Research and Therapy, 86, 35-49. https://doi.org/10.1016/j.brat.2016.07.007

King, K. M., Feil, M. C., \& Halvorson, M. A. (2018). Negative urgency is correlated with the use of reflexive and disengagement emotion regulation strategies. Clinical Psychological Science, 6(6), 822-834. https://doi.org/10.1177/2167702618785619

Klein, D. N., Ouimette, P. C., Kelly, H. S., Ferro, T., \& Riso, L. P. (1994). Test-retest reliability of team consensus best-estimate diagnoses of axis-I and axis-II disorders in a family study. American Journal of Psychiatry, 151, 1043-1047.

Kotov, R., Krueger, R. F., Watson, D., Achenbach, T. M., Althoff, R. R., Bagby, R. M., ... \& Eaton, N. R. (2017). The Hierarchical Taxonomy of Psychopathology (HiTOP): a dimensional alternative to traditional nosologies. Journal of abnormal psychology, 126(4), 454-477. https://doi.org/10.1037/abn0000258

Koval, P., Kalokerinos, E. K., Greenaway, K. H., Medland, H., Kuppens, P., Nezlek, J. B., ... Gross, J. (2020, August 16). Emotion Regulation in Everyday Life: What Can We Learn from Global Self-Reports? https://doi.org/10.31234/osf.io/cav54

Lavender, J. M., Tull, M. T., DiLillo, D., Messman-Moore, T., \& Gratz, K. L. (2017). 
Development and validation of a state-based measure of emotion dysregulation: The State Difficulties in Emotion Regulation Scale (S-DERS). Assessment, 24(2), 197-209. https://doi.org/10.1177/1073191115601218

Leyro, T. M., Zvolensky, M. J., \& Bernstein, A. (2010). Distress tolerance and psychopathological symptoms and disorders: a review of the empirical literature among adults. Psychological Bulletin, 136(4), 576. https://doi.org/10.1037/a0019712

Linehan, M. M. (2014). DBT Skills training manual (2 ${ }^{\text {nd }}$ ed.). Guilford Publications: New York. Liu, D. Y., \& Thompson, R. J. (2017). Selection and implementation of emotion regulation strategies in major depressive disorder: An integrative review. Clinical psychology review, 57, 183-194. https://doi.org/10.1016/j.cpr.2017.07.004

Lovibond, P. F., \& Lovibond, S. H. (1995). The structure of negative emotional states:

Comparison of the Depression Anxiety Stress Scales (DASS) with the Beck Depression and Anxiety Inventories. Behaviour Research and Therapy, 33(3), 335-343.

\section{https://doi.org/10.1016/0005-7967(94)00075-u}

Martin, R. C., \& Dahlen, E. R. (2005). Cognitive emotion regulation in the prediction of depression, anxiety, stress, and anger. Personality and Individual Differences, 39(7), 12491260. https://doi.org/10.1016/j.paid.2005.06.004

Naragon-Gainey, K., McMahon, T. P., \& Chacko, T. P. (2017). The structure of common emotion regulation strategies: A meta-analytic examination. Psychological Bulletin, 143(4), 384. https://doi.org/10.1037/bul0000093

Peters, J. R., Smart, L. M., \& Baer, R. A. (2015). Dysfunctional responses to emotion mediate the cross-sectional relationship between rejection sensitivity and borderline personality features. Journal of Personality Disorders, 29(2), 231-240.

https://doi.org/10.1521/pedi_2014_28_151 
Pfohl, B., Blum, N., St John, D., McCormick, B., Allen, J., \& Black, D. W. (2009). Reliability and validity of the Borderline Evaluation of Severity over Time (BEST): A self-rated scale to measure severity and change in persons with borderline personality disorder. Journal of Personality Disorders, 23, 281-293.

Pfohl, B., Blum, N., \& Zimmerman, M. (2009). Structured Interview for DSM-IV Personality (SIDP-IV). New York, NY: American Psychiatric Publishing.

Pisetsky, E. M., Haynos, A. F., Lavender, J. M., Crow, S. J., \& Peterson, C. B. (2017). Associations between emotion regulation difficulties, eating disorder symptoms, nonsuicidal self-injury, and suicide attempts in a heterogeneous eating disorder sample. Comprehensive Psychiatry, 73, 143-150. https://doi.org/10.1016/j.comppsych.2016.11.012

Pistorello, J., Hayes, S. C., Costello, E., Simpson, E., Begin, A., Rosen, K., \& Pearlstein, T. (2015). Suppression, acceptance, and monitoring of personally-relevant unwanted thoughts in women diagnosed with borderline personality disorder. Journal of Experimental Psychopathology, 6(4), 411-432. https://doi.org/10.5127/jep.042614

Prakash, R. S., Whitmoyer, P., Aldao, A., \& Schirda, B. (2017). Mindfulness and emotion regulation in older and young adults. Aging \& Mental Health, 21(1), 77-87. https://doi.org/10.1080/13607863.2015.1100158

Ruocco, A. C., Daros, A. R., Chang, J., Rodrigo, A. H., Lam, J., Ledochowski, J., \& McMain, S. F. (2019). Clinical, personality, and neurodevelopmental phenotypes in borderline personality disorder: a family study. Psychological Medicine, 49(12), 2069-2080. https://doi.org/10.1017/S0033291718002908

Salters-Pedneault, K., Roemer, L., Tull, M. T., Rucker, L., \& Mennin, D. S. (2006). Evidence of broad deficits in emotion regulation associated with chronic worry and generalized anxiety disorder. Cognitive Therapy and Research, 30(4), 469-480. https://doi.org/10.1007/s10608$\underline{006-9055-4}$ 
Selby E. A., Anestis, M. D., Bender, T. W., \& Joiner, T.E. Jr. (2009). An exploration of the emotional cascade model in borderline personality disorder. Journal of Abnormal Psychology, 118, 375-387. https://doi.org/10.1037/a0015711

Sloan, E., Hall, K., Moulding, R., Bryce, S., Mildred, H., \& Staiger, P. K. (2017). Emotion regulation as a transdiagnostic treatment construct across anxiety, depression, substance, eating and borderline personality disorders: A systematic review. Clinical Psychology Review, 57, 141-163. https://doi.org/10.1016/j.cpr.2017.09.002

Stepp, S. D., Scott, L. N., Morse, J. Q., Nolf, K. A., Hallquist, M. N., \& Pilkonis, P. A. (2014). Emotion dysregulation as a maintenance factor of borderline personality disorder features. Comprehensive Psychiatry, 55(3), 657-666.

\section{https://doi.org/10.1016/j.comppsych.2013.11.006}

Svaldi, J., Griepenstroh, J., Tuschen-Caffier, B., \& Ehring, T. (2012a). Emotion regulation deficits in eating disorders: A marker of eating pathology or general psychopathology? Psychiatry Research, 197(1-2), 103-111. https://doi.org/10.1016/j.psychres.2011.11.009

Svaldi, J., Dorn, C., Matthies, S., \& Philipsen, A. (2012b). Effects of suppression and acceptance of sadness on the urge for non-suicidal self-injury and self-punishment. Psychiatry Research, 200(2-3), 404-416. https://doi.org/10.1016/j.psychres.2012.06.030

Thompson, R. A. (2019). Emotion dysregulation: A theme in search of definition. Development and Psychopathology, 31, 805-815. https://doi.org/10.1017/S0954579419000282

Tobin, D. L., Holroyd, K. A., Reynolds, R. V., \& Wigal, J. K. (1989). The hierarchical factor structure of the Coping Strategies Inventory. Cognitive Therapy and Research, 13(4), 343361. https://doi.org/10.1007/BF01173478

Treynor, W., Gonzalez, R., \& Nolen-Hoeksema, S. (2003). Rumination reconsidered: A psychometric analysis. Cognitive Therapy and Research, 27(3), 247-259.

\section{https://doi.org/10.1023/A:1023910315561}


Vine, V., \& Aldao, A. (2014). Impaired emotional clarity and psychopathology: A transdiagnostic deficit with symptom-specific pathways through emotion regulation. Journal of Social and Clinical Psychology, 33(4), 319-342.

https://doi.org/10.1521/jscp.2014.33.4.319

Watson, D., Clark, L. A., \& Tellegen, A. (1988). Development and validation of brief measures of positive and negative affect: the PANAS scales. Journal of Personality and Social Psychology, 54(6), 1063-1070. https://doi.org/10.1037/0022-3514.54.6.1063

Wegner, D. M., \& Zanakos, S. (1994). Chronic thought suppression. Journal of Personality, 62(4), 615-640. https://doi.org/10.1111/j.1467-6494.1994.tb00311.x

Weiss, N. H., Gratz, K. L., \& Lavender, J. M. (2015). Factor structure and initial validation of a multidimensional measure of difficulties in the regulation of positive emotions: The DERSPositive. Behavior Modification, 39(3), 431-453. https://doi.org/10.1177/0145445514566504

Yoon, S., \& Rottenberg, J. (2020). Why do people with depression use faulty emotion regulation strategies? Emotion Review, 12(2), 118-128. https://doi.org/10.1177/1754073919890670

Zlomke, K. R., \& Hahn, K. S. (2010). Cognitive emotion regulation strategies: Gender differences and associations to worry. Personality and Individual Differences, 48(4), 408413. https://doi.org/10.1016/j.paid.2009.11.007 


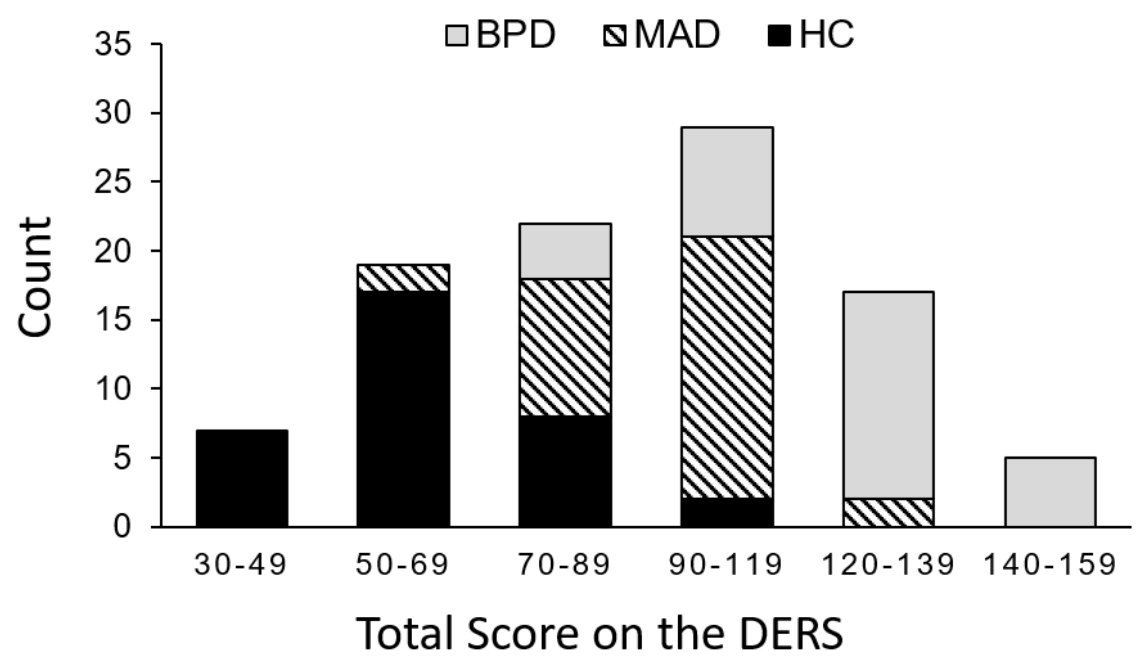

Figure 1. The distribution of total score on the DERS according to original recruitment group in Daros et al. (2018a). In the present study, an additional seven participants $(n=99)$ were included because of our transdiagnostic examination. $\mathrm{BPD}=$ Borderline personality disorder; $\mathrm{MAD}=$ Mixed anxiety and/or depression; $\mathrm{HC}=$ Healthy control. 
EMOTION REGULATION AND TRAIT EMOTION DYSREGULATION

Table 1. Results of the first principal components analysis reducing 25 variables from five selfreport measures into four component clusters of ER strategies.

\begin{tabular}{|c|c|c|c|c|c|c|}
\hline \multirow[t]{2}{*}{ Measure } & \multirow[t]{2}{*}{$\begin{array}{l}\text { ER Strategy Score/Subscale (Descriptor as per } \\
\text { documentation) }\end{array}$} & \multirow[t]{2}{*}{$\alpha$} & \multicolumn{4}{|c|}{$\begin{array}{c}\text { Components and factor } \\
\text { loadings }\end{array}$} \\
\hline & & & SRC1 & SRC2 & SRC3 & SRC4 \\
\hline CSI & Self-Criticism (blaming oneself for situation) & .95 & .89 & & & \\
\hline CERQ & Self-Blame (blaming oneself for experience) & .85 & .87 & & & \\
\hline CSI & Social Withdrawal (shutting off from others) & .93 & .77 & & & \\
\hline RRS & $\begin{array}{l}\text { Rumination (repetitive and passive focus on one's negative } \\
\text { thoughts) }\end{array}$ & .91 & .74 & & & \\
\hline WBSI & $\begin{array}{l}\text { Thought Suppression (tendency to suppress unwanted } \\
\text { thoughts) }\end{array}$ & .94 & .70 & & & \\
\hline MEAQ & $\begin{array}{l}\text { Repression/Denial (dissociation from or lack of awareness } \\
\text { of distress) }\end{array}$ & .87 & .68 & & & \\
\hline CSI & Wishful Thinking (reluctance to reframe) & .86 & .60 & & & \\
\hline CSI & Problem Solving (change stressful situation) & .84 & & .91 & & \\
\hline CSI & Cognitive Restructuring (alter the meaning) & .92 & & .87 & & \\
\hline CERQ & $\begin{array}{l}\text { Refocus on Planning (thoughts on how to handle } \\
\text { experience) }\end{array}$ & .83 & & .75 & & \\
\hline CERQ & $\begin{array}{l}\text { Positive Reappraisal (give a positive meaning to experience } \\
\text { in terms of personal growth) }\end{array}$ & .90 & & .73 & & \\
\hline MEAQ & Distress Endurance (effective behavior during distress) & .86 & & .67 & & \\
\hline CERQ & $\begin{array}{l}\text { Putting into Perspective (relativize negative event compared } \\
\text { to others) }\end{array}$ & .80 & & .59 & & \\
\hline CSI & Social Support (emotional support from others) & .91 & & .53 & & \\
\hline MEAQ & $\begin{array}{l}\text { Distraction/Suppression (attempts to ignore or suppress } \\
\text { distress) }\end{array}$ & .85 & & & -.73 & \\
\hline CERQ & Positive Refocus (thinking positive/pleasant thoughts) & .86 & & & -.69 & \\
\hline MEAQ & $\begin{array}{l}\text { Behavioral Avoidance (overt, situational avoidance of } \\
\text { distress) }\end{array}$ & .88 & & & -.63 & \\
\hline CERQ & Blame Others (blame others for experience) & .85 & & & & .82 \\
\hline CERQ & $\begin{array}{l}\text { Catastrophizing (thoughts emphasizing negativity of the } \\
\text { experience) }\end{array}$ & .81 & & & & .76 \\
\hline MEAQ & $\begin{array}{l}\text { Distress Aversion (negative attitudes or evaluations of } \\
\text { distress) }\end{array}$ & .91 & & & & .59 \\
\hline
\end{tabular}

Note : SRC = Self-report component; CSI = Coping Strategies Inventory; CERQ = Cognitive Emotion Regulation Questionnaire; RRS = Rumination and Response Style Questionnaire; WBSI = White Bear Suppression Inventory; MEAQ $=$ Multidimensional Experiential Avoidance Questionnaire; $\alpha=$ refers to Cronbach $\alpha$, a measure of internal consistency. The following variables were excluded from the principal components analysis because of low internal consistency (Cronbach $\alpha$ noted in brackets): CSI Express Emotions (.74; described as releasing and expressing emotions in documentation), CSI Problem Avoidance (.63; described as denial of problems/avoidance of thoughts), CERQ acceptance (.65; described as acceptance and resignation thoughts), and CERQ rumination (.62; described as having thoughts about the feelings and thoughts associated with negative events). The following variables were excluded because they did not load onto any component: MEAQ procrastination (.88; described as delaying impending distress). 
EMOTION REGULATION AND TRAIT EMOTION DYSREGULATION

Table 2. Results of the second principal components analysis reducing nine variables from the mood induction study into three component clusters of ER strategies.

\begin{tabular}{lcc}
\hline ER Strategy (Lay terms presented to participants) & $\begin{array}{c}\text { Components and factor } \\
\text { loadings } \\
\text { MIC2 }\end{array}$ & MIC3 \\
\hline Expressive Suppression (hiding your feelings from others) & .78 \\
Thought Avoidance (avoided thinking about it) & .74 \\
Thought Suppression (pushing down feelings or putting them out of mind) & .72 & .70 \\
Self-Criticism (criticizing self for feelings) & .69 \\
Acceptance (allowing or accepting your feelings) & .69 \\
Rumination (worrying or ruminating about the situation) & \\
$\begin{array}{l}\text { Cognitive Reappraisal (thinking of the situation differently to change how } \\
\text { you feel) }\end{array}$ & -.86 \\
$\begin{array}{l}\text { Problem Solving (coming up with ideas to change the situation or fix the } \\
\text { problem) }\end{array}$ & -.69 \\
$\begin{array}{l}\text { Note: } \text { MIC }=\text { Mood induction component; Experiential Avoidance (closing or averting eyes) was removed due to } \\
\text { lack of substantial loading. }\end{array}$
\end{tabular}


EMOTION REGULATION AND TRAIT EMOTION DYSREGULATION

Table 3. Linear regression predicting trait emotion dysregulation from resulting component clusters of ER strategies controlling for demographic and clinical variables.

\begin{tabular}{|c|c|c|c|c|c|}
\hline Step & Variable & $\beta$ & $t$ & $p$ & Model Fit and Test \\
\hline \multicolumn{6}{|c|}{ Self-Report } \\
\hline \multirow[t]{6}{*}{1} & Age & -.04 & -.56 & .58 & Model $F=30.79$ \\
\hline & Years of education & .07 & 1.08 & .28 & Model $p<.001$ \\
\hline & Ethnicity & -.04 & -.69 & .49 & $R^{2}=.68$ \\
\hline & Depression Symptoms & .21 & 1.93 & .06 & \\
\hline & Anxiety Symptoms & .17 & 1.90 & .06 & \\
\hline & BPD Symptoms & .56 & 5.82 & $<.001$ & \\
\hline \multirow[t]{10}{*}{2} & Age & -.07 & -1.33 & .19 & Model $F=37.78$ \\
\hline & Years of education & .02 & .41 & .69 & Model $p<.001$ \\
\hline & Ethnicity & .01 & .19 & .85 & $R^{2}=.82$ \\
\hline & Depression Symptoms & .02 & .17 & .87 & $\Delta R^{2}=.14, p<.001$ \\
\hline & Anxiety Symptoms & .06 & .85 & .40 & \\
\hline & BPD Symptoms & .35 & 4.31 & $<.001$ & \\
\hline & SRC1 & .46 & 6.48 & $<.001$ & \\
\hline & SRC2 & -.13 & -2.49 & .015 & \\
\hline & SRC3 & .03 & .57 & .57 & \\
\hline & SRC4 & .15 & 2.76 & .007 & \\
\hline \multicolumn{6}{|c|}{ Mood Induction } \\
\hline \multirow[t]{6}{*}{1} & Age & -.04 & -.57 & .57 & Model $F=31.56$ \\
\hline & Years of education & .07 & 1.05 & .30 & Model $p<.001$ \\
\hline & Ethnicity & -.05 & -.73 & .47 & $R^{2}=.68$ \\
\hline & Depression Symptoms & .20 & 1.90 & .06 & \\
\hline & Anxiety Symptoms & .18 & 1.98 & .051 & \\
\hline & BPD Symptoms & .56 & 5.86 & $<.001$ & \\
\hline \multirow[t]{9}{*}{2} & Age & -.06 & -.94 & .35 & Model $F=24.11$ \\
\hline & Years of education & .05 & .72 & .47 & Model $p<.001$ \\
\hline & Ethnicity & -.02 & -.31 & .76 & $R^{2}=.72$ \\
\hline & Depression Symptoms & .16 & 1.54 & .13 & $\Delta R^{2}=.04, p=.016$ \\
\hline & Anxiety Symptoms & .11 & 1.29 & .20 & \\
\hline & BPD Symptoms & .55 & 5.86 & $<.002$ & \\
\hline & $\mathrm{MIC1}$ & .23 & 3.22 & .002 & \\
\hline & MIC2 & -.05 & -.80 & .43 & \\
\hline & MIC3 & .08 & 1.19 & .24 & \\
\hline
\end{tabular}

Note: $\beta=$ standardized regression coefficient; $\mathrm{SRC}=$ Self-Report Component; $\mathrm{MIC}=$ Mood Induction Component. 
EMOTION REGULATION AND TRAIT EMOTION DYSREGULATION

Table 4. Partial correlations between facets of trait emotion dysregulation and component clusters of ER strategies controlling for demographic variables.

\begin{tabular}{lcccccc}
\hline DERS Subscale & Cronbach $\alpha$ & \multicolumn{5}{c}{ Partial correlation (controlling for demographic variables) } \\
& & \multicolumn{5}{c}{ with component number } \\
& & SRC1 & SRC2 & SRC4 & MIC1 & MIC3 \\
\hline Nonacceptance & .84 & $.71^{*}$ & -.18 & $.33^{*}$ & $.53^{*}$ & -.23 \\
Goals & .90 & $.53^{*}$ & -.24 & $.48^{*}$ & $.33^{*}$ & -.14 \\
Impulse & .92 & $.56^{*}$ & $-.41^{*}$ & $.48^{*}$ & $.42^{*}$ & -.20 \\
Awareness & .79 & $.57^{*}$ & $-.44^{*}$ & -.05 & .32 & .04 \\
Strategies & .94 & $.71^{*}$ & $-.49^{*}$ & $.58^{*}$ & $.42^{*}$ & -.23 \\
Clarity & .90 & $.60^{*}$ & $-.42^{*}$ & .08 & $.52^{*}$ & -.10 \\
\hline
\end{tabular}

Note SRC = Self-report component. MIC = Mood Induction component. Components SRC 3 and MIC2 were dropped from analyses as they were not significantly associated with trait emotion dysregulation as measured by the DERS total score. In this correlation matrix, a more stringent alpha was applied to determine statistical significance, therefore all asterisks refer to $p \mathrm{~s} \leq .001$. 